\title{
Considerations for a multiaxis nomenclature system for medical genetics
}

\author{
Nathaniel H. Robin, $M D^{1}$, and Leslie G. Biesecker, $M D^{2}$
}

Current diagnoses are based on a false dichotomy of phenotypic versus etiologic (molecular and environmental) factors. Some diagnostic labels rely solely on phenotypic factors and others rely on molecular or environmental factors. We are advocating consideration of a system that can encompass both phenotypic and etiologic factors and integrate these in a manner that is uniform and useful, both for the clinician and the researcher. Until recently, in the absence of a defining biochemical or genetic test, a syndrome or disorder was diagnosed by the observed constellation of signs and symptoms (including major and minor anomalies), and indirect laboratory tests that were consistently seen together in affected patients. Often the findings that distinguished syndromes were very subtle to all but the trained clinician, who could discern the relevant factors (clinical, molecular, and environmental) and reach a diagnosis (so called pattern recognition).

It was often impossible to know if these diagnostic designations accurately reflected the underlying biology of a given disorder or if they merely identified variations that represent pleiotropy. Advances in molecular technology have identified the molecular bases of many genetic syndromes and genetically determined diseases. For basic scientists, these discoveries have provided insight into the role of genes in both normal and abnormal human development and function. For clinicians, testing is now available to confirm a diagnosis and to permit prenatal testing. However, these discoveries have also provided information that has challenged the definition of a genetic syndrome. ${ }^{1-3}$ For example, some disorders that appeared clinically distinct were found to be allelic and other disorders that appeared clinically homogenous or consistent demonstrated locus heterogeneity. In some instances, molecular advances have rendered the clinical or molecular nomenclature alone unable to accurately describe a phenotype in a particular patient.

One example of this is the craniosynostosis syndromes Apert, Pfeiffer, Crouzon, Jackson-Weiss, and Saethre-Chotzen, which demonstrate both genetic heterogeneity and pleiotropy. The Pfeiffer syndrome phenotype can be caused by mutations in the genes FGFR1, 2, or 3. Similarly, the Saethre-Chotzen

From the ${ }^{1}$ Departments of Genetics, Pediatrics, Otolaryngology, and Reproductive Biology, Case Western Reserve University School of Medicine, University Hospitals of Cleveland, Cleveland, Ohio, ${ }^{2}$ National Human Genome Research Institute, NIH, Bethesda, Maryland. Nathaniel H. Robin, MD, Center for Human Genetics, Lakeside 1500, Cleveland, OH 44106-6506.

Leslie G. Biesecker, MD, National Institutes of Health, National Human Genome Research Institute, 49 Convent Drive Room 4A80, Bethesda, MD 20892-4472.

Received: January 16, 2001.

Accepted: February 28, 2001. phenotype can be caused by mutations in TWIST or FGFR3. Apert, Crouzon, Pfeiffer, Jackson-Weiss syndromes are each clinically distinct entities (albeit with some phenotypic overlap), yet all are caused by FGFR2 mutations. ${ }^{3-5}$ There is no shortage of additional examples of this problem. Hirschsprung disease, ${ }^{6}$ tuberous sclerosis, ${ }^{7}$ and Bardet-Biedl syndrome ${ }^{8}$ are all phenotypically defined conditions that demonstrate genetic locus heterogeneity. An example of allelic pleiomorphic diversity is GLI3, in which various allelic mutations cause Grieg cephalopolysyndactyly syndrome, Pallister-Hall syndrome, and postaxial polydactyly type A. ${ }^{9}$ These examples demonstrate that consideration of either phenotypic or etiologic descriptors alone is either inadequate or represents conflicting approaches to the generation of diagnostic labels for affected patients. To address this issue, we propose that both phenotypic and etiologic factors should be used to generate a unified diagnostic labeling system. We begin by outlining the underlying assumptions of this approach and then delineate a proposed system.

\section{THE UNDERLYING ASSUMPTIONS}

We suggest that the following principles should guide development of a valid diagnostic schema.

1. Clinical criteria and phenotypic descriptors are relevant and useful independent of etiologic descriptors.

2. Etiologic factors or descriptors are relevant and useful independent of clinical descriptors.

3. Therefore, clinical and etiologic criteria are equally important and valid and should be integrated.

4. Current knowledge suggests that etiologic factors include genetic and environmental factors.

5. The system must be able to describe single gene disorders as well as multifactorial and complex genetic traits.

6. The system must be flexible and amenable to change as knowledge evolves.

7. The system must allow designations to describe patients with partially characterized phenotypes.

8. The system should be easy to use.

\section{THE PROPOSED SYSTEM}

This proposed nomenclature system should be regarded as a starting point for wide-ranging discussion of these issues. We delineate below a system that incorporates clinical, molecular genetic, and environmental findings or factors as three separate categories. It is modeled after the "diagnostic axis" system 
used in the Diagnostic and Statistical Manual V. The proposed system has three main diagnostic axes:

- Axis I describes the clinical diagnosis, or the clinical phenotype.

- Axis II describes the underlying molecular genetic $\operatorname{defect}(\mathrm{s})$.

- Axis III describes the environmental or nongenetic factor(s).

For example, a male child with the clinical findings of Down syndrome in whom cytogenetic testing revealed trisomy for chromosome 21 would be written as:

Axis I: Down syndrome

Axis II: 47,XY, +21

Axis III: N/A

This designation makes explicit that the patient has a set of recognizable physical features that warrant a label of Down syndrome, irrespective of whether he has $47, \mathrm{XY},+21$ or $46, \mathrm{XY}$, iso21q, or $46, \mathrm{XY},-13,+13 \mathrm{q}: 21 \mathrm{q}$, or any other cytogenetic aberration that yields trisomy for all or most of chromosome 21q. Furthermore, it reflects current opinion that environmental factors do not play a significant role in the determination of the phenotype relative to that of the cytogenetic aberration.

Another example: a macrosomic male infant with an omphalocele, macroglossia, and hemihyperplasia for whom cytogenetics and UPD 11 testing were both normal would be designated:

Axis I: Beckwith-Wiedemann syndrome

Axis II: 46,XY,non-UPD11

Axis III: N/A

This example includes negative results. We propose that such results are useful if they directly bear on the primary diagnosis for the patient. In contrast, we would not include a normal karyotypic result for a patient with cystic fibrosis because current understanding is that a karyotypic aberration is rarely, if ever, associated with CF. If a specific mutation in the IGF2 gene were subsequently detected in the patient with Beckwith-Wiedemann syndrome, the Axis II designation could be modified accordingly. This again is an explicit recognition that the patient and the clinical phenotype that she manifests are not necessarily altered based on the result of a molecular test.

Designation of disease phenotypes that exhibit locus heterogeneity, such as retinitis pigmentosa (RP), ${ }^{10}$ will be straightforward to accurately classify and describe in this system. The Axis I designation would simply be "retinitis pigmentosa," followed by the clinical description that has implications as to underlying diagnosis, such as age-of-onset. Axis II would list the RP genes that had been tested. If the genetic testing has failed to reveal the etiology of the RP, the genes that have tested negative would be listed.

Axis I: Retinitis pigmentosa, autosomal dominant inheritance, age of onset 15 years
Axis II: RHO negative, Peripherin negative

Axis III: N/A

We recognize that the description of negative data may be a reflection of test methodology rather than the molecular biology of the patient. It is well recognized that all methodologies for determining molecular alterations are $<100 \%$ sensitive. However, this is no different than a nonmolecular test result, as any test can have a false-negative result due to technical errors, sample mix-ups, limitations of the test, etc. We propose that the system simply represent the best assessment of the molecular testing by the molecular diagnostic laboratory and the clinician caring for the patient.

The above example includes modifiers of the Axis I specification (phenotype) that specify phenotypic findings that are recognized to have important clinical implications and which are associated with etiologic factors. For example, earlier age of onset of retinitis pigmentosa reduces the probability that the phenotype is caused by a mutation in the peripherin gene. In addition, the inheritance pattern excludes a number of genes that are known to cause retinitis pigmentosa inherited in an autosomal recessive (or digenic) or X-linked pattern. These modifiers are simply listed serially following the primary diagnosis in the above example but could also be recorded as subheadings. Alternatively, the above patient could be described as:

Axis I: Retinitis pigmentosa

I.A: Autosomal dominant inheritance

I.B: Age of onset 15 years

Axis II: RHO negative, Peripherin negative

Axis III: N/A

The purpose of these modifiers is to permit the listing of relevant phenotypic details that are not necessarily implied in the primary Axis I designation. Additional modifiers should not be used just to add clinical details about a specific patient. For example, the listing of "visual acuity 20/200" would be an inappropriate use of the system for the above patient, because it is not a feature that delineates a recognized clinical subtype of retinitis pigmentosa. These modifiers should instead be used for data that have been shown to have relevance to the basic underlying cause of the disease. For example, for an obese male with hypogonadism, hypopigmentation, short stature, and developmental delay, the diagnosis would be:

Axis I: Prader-Willi syndrome

I.A. Hypopigmentation

Axis II: Non-UPD 15, nondeleted for SNRPN FISH probe

Axis III: N/A

In this case, the presence of hypopigmentation implies that the genetic defect is a deletion, rather than UPD. ${ }^{11}$ The advantages and disadvantages of specifying these phenotypic descriptors as either strings of features or as subheadings are primarily a trade-off of complexity and formality versus ease of use. 
This system should be adaptable to multifactorial traits and complex genetic disorders. The challenge here is that none of these disorders are sufficiently understood to know how many factors may be involved in these conditions and how those factors will be evaluated, analyzed, and presented. Below is an example for disease " $\mathrm{X}$," for which several causative genes have been identified ( $A, B, C, D$ and $E$ ) where the genes have a disease-associated "abnormal" allele and a protective "normal" allele. We assume, for simplicity that the five loci have an additive or cumulative effect and do not interact.

Axis I: Complex genetic trait " $\mathrm{X}$ "

Axis II: Gene "A" negative, gene "B" negative, gene " $C$ " negative, gene " $D$ " positive, gene "E" negative

Axis III: N/A

Alternatively, such disorders may be found to have multiple factors that influence susceptibility in a complex, interactive manner. In such a case, complex modeling of genotypes at many loci may be required to assess relative risk, much as consideration of MSAFP, estriol, $\beta \mathrm{HCG}$, and maternal age are used simultaneously to derive a relative risk for fetal trisomy. In this case, axes II and III are collapsed to yield an aggregate relative risk factor.

Axis I: Uncomplicated pregnancy

Axes II/III: Triple screen/maternal age relative risk for trisomy: 1.60

The same would apply for modifier genes in a complex genetic trait. For example, it is becoming clear that cystic fibrosis (CF) should not be considered a single gene disorder, but one that is modified by other as yet unidentified genes. ${ }^{12,13}$ The proposed diagnostic system will permit clinicians and clinical researchers to describe a given patient's disorder more thoroughly. The example below describes a patient with CF who has severe pulmonary disease but mild gastrointestinal (GI)pathology. Modifier gene A (associated with severe pulmonary disease) and modifier gene "B" (associated with mild GI disease) are tested for.

Axis I: Cystic fibrosis, severe pulmonary disease, mild gastrointestinal disease

Axis II: $\Delta$ F508/unknown, pulmonary modifier "A" positive, GI modifier " $B$ " negative

Axis III: N/A

The importance of "environmental factors" will continue to grow as we increase our understanding of the relationship of genetic and nongenetic factors to the expression of a given phenotype and genotype. To date, only a few environmental factors for multifactorial traits have been identified, but we can expect numerous examples in the future. Therefore, "environmental factor(s)" deserve a separate Axis, signifying their importance. One presently identified example of this is aminoglycoside-induced hearing loss and the mitochondrial A1555G mutation. ${ }^{14}$ Here, both the primary genetic determinant (the mitochondrial tRNA mutation) and the primary environmental factor (exposure to an aminoglycoside antibiotic) are known. Below is an example of how the system would be used to describe a patient with these findings.

Axis I: Progressive sensorineural hearing loss

Axis II: Mitochondrial A1555G mutation positive

Axis III: Aminoglycoside exposure

\section{DISCUSSION}

The field of medical genetics is entering a new era, one in which the genetic determinants are being identified not only for rare genetic syndromes but for common diseases, such as hypertension and diabetes, as well. The purpose of this proposal is to initiate discussion of a systematic approach to disease nomenclature that incorporates the clinical or phenotypic diagnosis and the relevant etiologic factors, be they genetic or environmental. The arenas of clinical medicine and human molecular genetics have distinct and complementary views of genotype-phenotype interactions. Most clinicians and scientists should be expected to use the knowledge with which they are most familiar to describe patients and disorders. For these reasons, competing and conflicting notions of description and classification will arise. The most common manifestation of this issue is that some advocate a molecular label for disorders to replace the phenotypic label. We reject this notion because it does not take into account clear evidence of gene-environment interactions and it does not acknowledge the critical importance of phenotype. In the clinic, clinicians must care for pa-

Table 1

Incorporating the OMIM system

\begin{tabular}{|c|c|c|c|}
\hline \multicolumn{2}{|l|}{ Axis I } & \multicolumn{2}{|c|}{ Axis II } \\
\hline Phenotype name & Phenotype OMIM & Gene name & Gene OMIM \\
\hline \multirow[t]{3}{*}{ Pfeiffer syndrome } & \multirow[t]{3}{*}{$\# 101600$} & FGFR1 & \#136356 \\
\hline & & FGFR2 & \#176943 \\
\hline & & FGFR3 & \#134934 \\
\hline \multirow[t]{3}{*}{ Saethre-Chotzen syndrome } & $\# 101400$ & TWIST & \#601622 \\
\hline & & FGFR3 & \#134934 \\
\hline & & FGFR2 & \#176943.0023 \\
\hline Apert syndrome & \#101200 & \multirow[t]{4}{*}{ FGFR2 } & \multirow[t]{4}{*}{ \#176943 } \\
\hline Crouzon syndrome & \#123500 & & \\
\hline Pfeiffer syndrome & \#101600 & & \\
\hline Jackson-Weiss syndrome & $\# 123150$ & & \\
\hline OI type I & $\# 166200$ & \multirow{4}{*}{$\begin{array}{r}\text { COL1A1 or } \\
\text { COL1A2 }\end{array}$} & \multirow{4}{*}{$\begin{array}{r}\# 120150 \text { or } \\
\# 120160\end{array}$} \\
\hline OI type II & \#166210 & & \\
\hline OI type III & \#259420 & & \\
\hline OI type IV & $\# 166220$ & & \\
\hline
\end{tabular}


tients, not alleles, risk factors, or abstract concepts. For this reason, we believe that the phenotype descriptor should always be part of the system to describe patients. This system should be taken as an explicit rejection of any dichotomous characterization of gene-environment interactions or genotype-phenotype correlation. Instead, it is an acknowledgment that phenotypes are related to genes and environment in a complex and sometimes subtle manner.

Weaknesses of this proposal are that there is no uniform method for describing the phenotype of many disorders and there is little experience in describing environmental exposures. The OMIM system provides the best existing framework for phenotypic descriptors and mutations. Table 1 illustrates how the OMIM system could be incorporated into the proposed nomenclature system. However, by itself, the OMIM system is limited and does not address several of our assumptions regarding an ideal nomenclature. Some of the phenotypic descriptors are tied to genetic locus descriptions, even when there are little or no data to suggest associations of phenotypic subtypes with these loci (e.g., Bardet-Biedl syndrome 1, 2, etc.). In contrast, there are clear schemes for the description of both cytogenetic and molecular genomic alterations. Both of these schemes can be used without alteration as entries in Axis II.

Another weakness is that the proposed system may require integration with ICD10 coding schemes, the primary diagnostic coding scheme for billing and reimbursement. Another coding system, SNOMED, has been developed as a chart coding scheme with 17 axes and thousands of specified terms. ${ }^{15}$ The extreme complexity of this system makes it powerful and flexible for coding charts, but this complexity and low acceptance make it unwieldy for use by clinicians. ${ }^{16}$

The system proposed in the present paper should be straightforward to integrate into any of these other systems as it has a clear logical basis and independently identifies relevant clinical factors. Our intention was to generate the simplest structured framework that systematically describes phenotypes and relevant etiologic factors to facilitate research and medical care. While we appreciate that this system may not solve all of the issues that we have highlighted, we believe it represents a beginning point for developing a new nomenclature system that is needed as medical genetics enters this new era.

\section{References}

1. Lurie IW, Opitz JM. Phenotypic mapping and clinical ideology. Am J Med Genet 1995;57:587.

2. Biesecker L. Lumping and splitting: molecular biology in the genetics clinic. Clin Genet 1998;53:3-7.

3. Cohen MM Jr, Maclean RE. Should syndromes be defined phenotypically or molecularly? Resolution of the dilemma [editorial]. Am J Med Genet 1999;86:203-204.

4. Robin NH. Molecular genetic advances in craniosynostosis. Plast Reconstr Surg 1999;103:1060-1070.

5. Hehr U, Muenke M. Craniosynostosis syndromes: from genes to premature fusion of skull bones. Mol Gen Metab 1999;68:139-151.

6. Kusafuka T, Puri P. Genetic aspects of Hirschsprung's disease. Semin Pediatr Surg 1998;7:148-155.

7. Jones AC, Shyamsundar MM, Thomas MW, Maynard J, Idziaszcyk S, Tomkins S, Sampson JR, Cheadle JR. Comprehensive mutation analysis of TSC1 and TSC2-and phenotypic correlations in 150 families with tuberous sclerosis. Am J Hum Genet 1999;64:1305-1315.

8. Beals PL, Elcioglu N, Woolf AS, Parker D, Flinter FA. New criteria for improved diagnosis of Bardet-Biedl syndrome: results of a population survey. J Med Genet 1999;36:437-446.

9. Biesecker L. Strike three for GLI3. Nat Genet 1997;17:259-260.

10. Phelan JK, Bok D. A brief review of retinitis pigmentosa and the identified retinitis pigmentosa genes. Mol Vis 2000;6:116-124.

11. Cassidy SB, Forsythe M, Heeger S, Nicholls RD, Schork N, Benn P, Schwartz S. Comparison of phenotype between patients with Prader-Willi syndrome due to deletion 15q and uniparental disomy 15. Am J Med Genet 1997;68:433-440.

12. Garred P, Pressler T, Madsen HO, Frederiksen B, Svejgaard A, Hoiby N, Schwartz M, Koch C. Association of mannose-binding lectin gene heterogeneity with severity of lung disease and survival in cystic fibrosis. J Clin Invest 1999;104:431-437.

13. Zielenski J, Corey M, Rozmahel R, Markiewicz D, Aznarez I, Casals T, Larriba S, Mercier B, Cutting GR, Krebsova A. Detection of a cystic fibrosis modifier locus for meconium ileus on human chromosome 19q13. Nat Genet 1999;22:128-129.

14. Fischel-Ghodsian N. Genetic factors in aminoglycoside toxicity. Ann N Y Acad Sci 1999;884:99-109.

15. Lussier YA, Rothwell DJ, Cote RA. The SNOMED model: a knowledge source for the controlled terminology of the computerized patient record. Methods Inf Med 1998;37:161-164.

16. Baldwin G. A flurry of terms, but not many users. Health Data Manag 2000;8:52-60. 